\title{
The Different Strategies used for the Early Diagnosis of Alzheimer's Disease
}

\author{
C. S. Sandeep ${ }^{1}$ and A. Sukesh Kumar ${ }^{2}$ \\ ${ }^{1 \& 2}$ Department of ECE, College of Engineering, University of Kerala, Kerala, India \\ E-Mail: sandeepcs07nta@gmail.com,drsukeshkumar@yahoo.in
}

\begin{abstract}
Gerontology or the scientific study of old age deal with the many clinical problems that are common in the elderly population and many of these follow the orthodox pattern of clinical practice. Patients characteristically have poor insight and often attribute their early symptoms of amnesia to normal aging. Alzheimer's disease (AD) is a common form of senile dementia that makes disabilities in cognitive behavior and performs routine functions. There are several causes for the disease. Although our understanding of the key steps underlying neurodegeneration in Alzheimer's disease $(\mathrm{AD})$ is incomplete, it is clear that it begins long before symptoms are noticed by the patient. The aim of this paper is to give an overall idea of the hallmarks, stages of the disease, signs or symptoms and the different methods used for its diagnosis. Any disease-modifying treatments which are developed are most likely to be successful if initiated early in the process, and this requires that we develop reliable, validated and economical ways to diagnose Alzheimer's-type pathology. However, despite comprehensive searches, no single test has shown adequate sensitivity and specificity, and it is likely that a combination will be needed. There are several clinical tests and neuroimaging techniques used in clinical practice for the diagnosis of Alzheimer's - type pathology. Prominent of them are biomarkers, Magnetic Resonance Imaging Scan (MRI), Positron Emission Tomography (PET) and Single-Proton CT Scanning (SPECT). Using the new advanced Biomedical Engineering Technologies to the clinical practices stated above, we can develop a computer-aided tool for the early diagnosis of AD. The different soft computing tools in Biomedical Engineering for developing a computeraided tool are Neural Networks, Genetic algorithm, Wavelet Networks, Support Vector Machines, and Fuzzy Logic. In this paper, we have focused on the different causes as well as the different strategies used for the early diagnosis of Alzheimer's disease (AD).
\end{abstract}

Keywords: Alzheimer's Disease, Gerontology, Early Diagnosis, Biomarkers, Neuroimaging

\section{INTRODUCTION}

In the past several decades, investigators have learned much about what happens in the brain when people have a neurodegenerative disease such as Parkinson's disease, $\mathrm{AD}$, or other dementias. Their findings also have revealed much about what happens during healthy aging. Researchers are investigating a number of changes related to healthy aging in hopes of learning more about this process so they can fill gaps in our knowledge about the early stages of $\mathrm{AD}$ [1]. As a person gets older, changes occur in all parts of the body, including the brain. Certain parts of the brain shrink especially the prefrontal cortex (an area at the front of the frontal lobe) and the hippocampus. Both areas are important to learning, memory, planning, and other complex mental activities. Changes in neurons and neurotransmitters affect communication between neurons. In certain brain regions, communication between neurons can be reduced because white matter (myelin-covered axons) is degraded or lost [2]. Changes in the brain's blood vessels occur. Blood flow can be reduced because arteries narrow and less growth of new capillaries occurs. In some people, structures called plaques and tangles develop outside of and inside neurons, respectively, although in much smaller amounts than in $\mathrm{AD}$. It also appears that additional brain regions can be activated in older adults during cognitive tasks, such as taking a memory test. Researchers do not fully understand why this happens, but one idea is that the brain engages mechanisms to compensate for difficulties that certain regions may be having. For example, the brain may recruit alternate brain networks in order to perform a task [3]. These findings have led many scientists to believe that major declines in mental abilities are not as inevitable as people age. Growing evidence of the adaptive (what scientists call "plastic") capabilities of the older brain provide hope that people may be able to do things to sustain good brain function as they age [4]. A variety of interacting factors, such as lifestyle, overall health, environment, and genetics also may play a role [5].

\section{THE HALLMARKS OF AD}

Alzheimer's disease disrupts critical metabolic processes that keep neurons healthy. These disruptions cause nerve cells in the brain to stop working, lose connections with other nerve cells, and finally die. The destruction and death of nerve cells cause memory failure, personality changes, problems in carrying out daily activities, and other features of the disease [6]. The brains of people with AD have an abundance of two abnormal structures-amyloid plaques and neurofibrillary tangles - that are made of misfolded. This is especially true in certain regions of the brain that are important in memory [8]. The third main feature of $\mathrm{AD}$ is the loss of connections between cells. This leads to diminished cell function and cell death.

\section{A. Amyloid Plaques}

Amyloid plaques are found in the spaces between the brain's nerve cells. They were first described by Dr.Alois 
Alzheimer in 1906. Plaques consist of largely insoluble deposits of an apparently toxic protein peptide, or fragment called amyloid. We now know that some people develop some plaques in their brain tissue as they age. However, the $\mathrm{AD}$ brain has many more plaques in particular brain regions. We still do not know whether amyloid plaques themselves cause AD or whether they are a by-product of the AD process [9]. We do know that genetic mutations can increase production of beta-amyloid and can cause rare, inherited forms of AD. Amyloid precursor protein is the starting point for amyloid plaques, is one of many proteins associated with the cell membrane, the barrier that encloses the cell. As it is being made inside the cell, APP becomes embedded in the membrane, as a toothpick stuck through the skin of an orange in Fig.1.

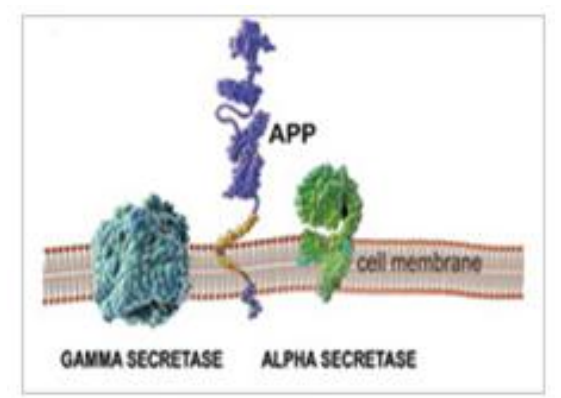

Fig. 1 APP becomes embedded in the membrane

In a number of cell compartments, including the outermost cell membrane, specific enzymes snip, or cleave, APP into discrete fragments. In 1999 and 2000, scientists identified the enzymes responsible for cleaving APP [10]. These enzymes are called alpha-secretase, beta-secretase, and gamma-secretase. In a major breakthrough, scientists then discovered that, depending on which enzyme is involved and the segment of APP where the cleaving occurs, APP processing can follow one of two pathways that have very different consequences for the cell [11].

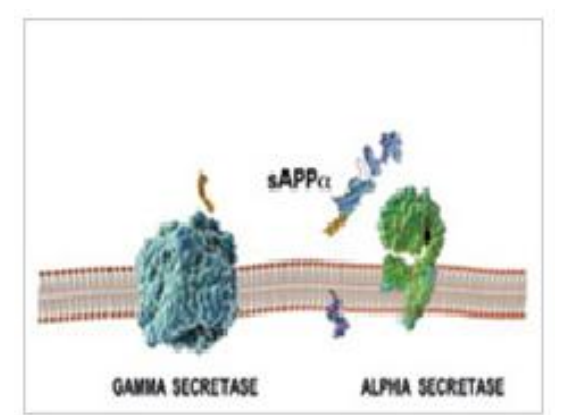

Fig. 2 The larger fragment remains within the neuron and interacts with factors in the nucleus

In the beginning pathway, alpha-secretase cleaves the APP molecule within the portion that has the potential to become beta-amyloid. This eliminates the production of the betaamyloid peptide and the potential for plaque buildup. The cleavage releases from the neuron a fragment called sAPP $\alpha$, which has beneficial properties, such as promoting neuronal growth and survival. The remaining APP fragment, still tethered in the neuron's membrane, is then cleaved by gamma-secretase at the end of the beta-amyloid segment. The smaller of the resulting fragments also is released into space outside the neuron, while the larger fragment remains within the neuron and interacts with factors in the nucleus as in Figure 2. In the harmful pathway, beta-secretase first cleaves the APP molecule at one end of the beta-amyloid peptide, releasing sAPP $\beta$ from the cell in Figure 3. Gammasecretase then cuts the resulting APP fragment, still tethered in the neuron's membrane, at the other end of the betaamyloid peptide [12]. Following the cleavages at each end, the beta-amyloid peptide is released into space outside the neuron and begins to stick to other beta-amyloid peptides in Figure 4. These small, soluble aggregates of two, three, four, or even up to a dozen beta-amyloid peptides are called oligomers. Specific sizes of oligomers may be responsible for reacting with receptors on neighboring cells and synapses, affecting their ability to function [13].

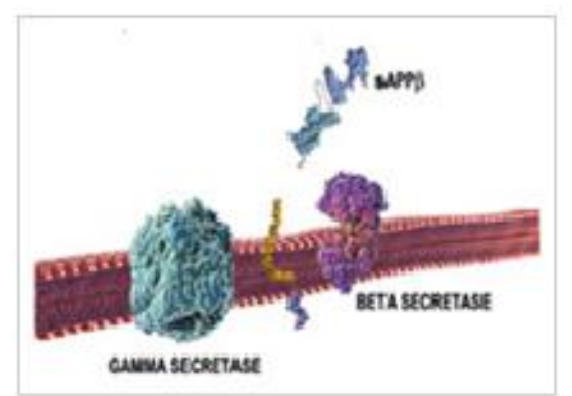

Fig. 3 Beta-secretase first cleaves the APP molecule at one end of the beta-amyloid peptide, releasing sAPP $\beta$ from the cell

It is likely that some oligomers are cleared from the brain. Those that cannot be cleared clump together with more beta-amyloid peptides. As the process continues, oligomers grow larger, becoming entities called protofibrils and fibrils. Eventually, other proteins and cellular material are added, and these increasingly insoluble entities combine to become the well-known plaques that are characteristic of AD [14].

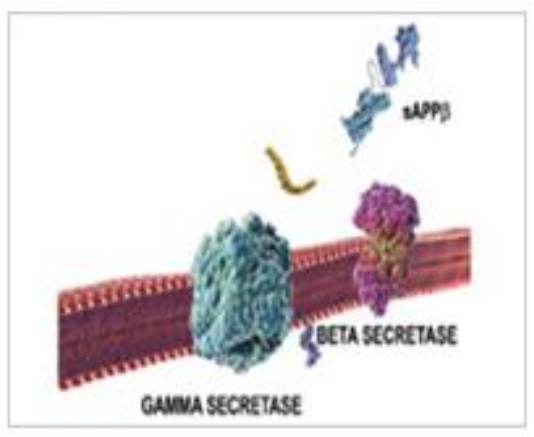

Fig. 4 The beta-amyloid peptide is released into the space outside the neuron and begins to stick to other beta-amyloid peptides

For many years, scientists thought that plaques might cause all of the damage to neurons that is seen in AD. However, that concept has evolved greatly in the past few years. Many scientists now think that oligomers may be a major culprit. Many scientists also think that plaques actually may be a late-stage attempt by the brain to get this harmful betaamyloid away from neurons [15]. 


\section{B. Neurofibrillary Tangles}

The second hallmark of $\mathrm{AD}$, also described by Dr. Alzheimer, is neurofibrillary tangles. Tangles are abnormal collections of twisted protein threads found inside nerve cells. The chief component of tangles is a protein called tau [16]. Healthy neurons are internally supported in part by structures called microtubules, which help transport nutrients and other cellular components, such as neurotransmitter-containing vesicles, from the cell body down the axon. Tau, which usually has a certain number of phosphate molecules attached to it, binds to microtubules and appears to stabilize them. In $\mathrm{AD}$, an abnormally large number of additional phosphate molecules attach to tau. As a result of this "hyperphosphorylation," tau disengages from the microtubules and begins to come together with other tau threads. These tau threads form structures called paired helical filaments, which can become enmeshed with one another, forming tangles within the cell. The microtubules can disintegrate in the process, collapsing the neuron's internal transport network. This collapse damages the ability of neurons to communicate with each other [17].

\section{Loss of Connection between Cells and Cell Death}

The third major feature of $\mathrm{AD}$ is the gradual loss of connections between neurons. Neurons live to communicate with each other, and this vital function takes place at the synapse. Since the 1980s, new knowledge about plaques and tangles has provided important insights into their possible damage to synapses (figure 5) and on the development of $\mathrm{AD}$. The $\mathrm{AD}$ process not only inhibits communication between neurons but can also damage neurons to the point that they cannot function properly and eventually die. As neurons die throughout the brain, affected regions begin to shrink in a process called brain atrophy. By the final stage of $\mathrm{AD}$, the damage is widespread, and brain tissue has shrunk significantly [18].

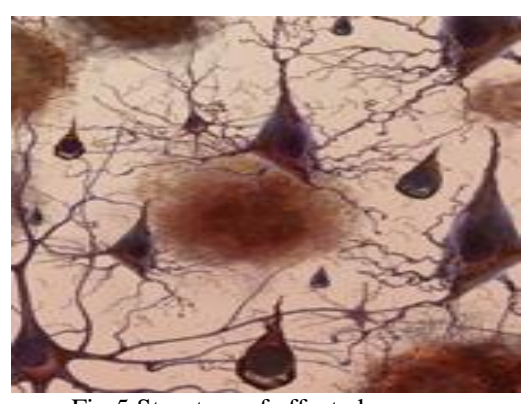

Fig.5 Structure of affected neurons

\section{THE CHANGING BRAIN IN AD}

No one knows exactly what starts the AD process or why some of the normal changes associated with aging become so much more extreme and destructive in people with the disease. We know a lot, however, about what happens in the brain once AD takes hold and about the physical and mental changes that occur over time. The time from diagnosis to death varies - as little as 3 or 4 years if the person is older than 80 when diagnosed to as long as 10 or more years if the person is younger. Several other factors besides age also affect how long a person will live with AD. These factors include the person's sex, the presence of other health problems, and the severity of cognitive problems at diagnosis. Although the course of the disease is not the same in every person with $\mathrm{AD}$, symptoms seem to develop over the same general stages.

\section{A. Preclinical $A D$}

$\mathrm{AD}$ begins deep in the brain, in the entorhinal cortex, a brain region that is near the hippocampus and has direct connections to it. Healthy neurons in this region begin to work less efficiently, lose their ability to communicate, and ultimately die. This process gradually spreads to the hippocampus, the brain region that plays a major role in learning and is involved in converting short-term memories to long-term memories [19]. Affected regions begin to atrophy. Ventricles, the fluid-filled spaces inside the brain, begin to enlarge as the process continues. Scientists believe that these brain changes begin 10 to 20 years before any clinically detectable signs or symptoms of forgetfulness appear. That's why they are increasingly interested in the very early stages of the disease process. They hope to learn more about what happens in the brain that sets a person on the path to developing $\mathrm{AD}$. By knowing more about the early stages, they also hope to be able to develop drugs or other treatments that will slow or stop the disease process before significant impairment occurs.

\section{OTHER SIGNS OF EARLY AD DEVELOPMENT}

As scientists have sharpened their focus on the early stages of $\mathrm{AD}$, they have begun to see hints of other changes that may signal a developing disease process. For example, in the Religious Orders Study, a large AD research effort that involves older nuns, priests, and religious brothers, investigators have explored whether changes in older adults' ability to move about and use their bodies might be a sign of early $\mathrm{AD}$. The researchers found that participants with MCI had more movement difficulties than the cognitively healthy participants but less than those with AD [20]. Moreover, those with MCI who had lots of trouble moving their legs and feet were more than twice as likely to develop $\mathrm{AD}$ as those with good lower body function. It is not yet clear why people with MCI might have these motor function problems, but the scientists who conducted the study speculate that they may be a sign that damage to blood vessels in the brain or damage from $\mathrm{AD}$ is accumulating in areas of the brain responsible for motor function. If further research shows that some people with MCI do have motor function problems in addition to memory problems, the degree of difficulty, especially with walking, may help identify those at risk of progressing to AD. Other scientists have focused on changes in sensory abilities as possible indicators of early cognitive problems. For example, in one study they found associations between a decline in the ability to detect odours and cognitive problems or dementia. 
These findings are tentative, but they are promising because they suggest that, someday, it may be possible to develop ways to improve early detection of MCI or AD. These tools also will help scientists answer questions about causes and very early development of $\mathrm{AD}$, track changes in brain and cognitive function over time, and ultimately track a person's response to treatment for $\mathrm{AD}$.

\section{A. Mild AD}

As AD spreads through the brain, the number of plaques and tangles grows, shrinkage progresses, and more and more of the cerebral cortex is affected. Memory loss continues and changes in other cognitive abilities begin to emerge. The clinical diagnosis of $\mathrm{AD}$ is usually made during this stage. Signs of mild AD can include:

1. Memory loss

2. Confusion about the location of familiar places (getting lost begins to occur)

3. Taking longer than before to accomplish normal daily tasks

4. Trouble handling money and paying bills

5. Poor judgment leading to bad decisions

6. Loss of spontaneity and sense of initiative

7. Mood and personality changes, increased anxiety and/or aggression

In mild $\mathrm{AD}$, a person may seem to be healthy but is actually having more and more trouble making sense of the world around him or her. The realization that something is wrong often comes gradually to the person and his or her family. Accepting these signs as something other than normal and deciding to go for diagnostic tests can be a big hurdle for people and families. Once this hurdle is overcome, many families are relieved to know what is causing the problems. They also can take comfort in the fact that despite a diagnosis of MCI or early $\mathrm{AD}$, a person can still make meaningful contributions to his or her family and to society for a time [21].

\section{B. Moderate $A D$}

By this stage, AD damage has spread to the areas of the cerebral cortex that control language, reasoning, sensory processing, and conscious thought. Affected regions continue to shrink, ventricles enlarge, and signs and symptoms of the disease become more pronounced and widespread. Behavioral problems, such as wandering and agitation, can occur. More intensive supervision and care become necessary, which can be difficult for many spouses and families. The symptoms of this stage can include:

1. Increasing memory loss and confusion

2. Shortened attention span

3. Inappropriate outbursts of anger

4. Problems recognizing friends and family members

5. Difficulty with language and problems with reading, writing, and working with numbers

6. Difficulty organizing thoughts and thinking logically
7. Inability to learn new things or to cope with new or unexpected situations

8. Restlessness, agitation, anxiety, tearfulness, wandering - especially in the late afternoon or at night

9. Repetitive statements or movement, occasional muscle twitches

10. Hallucinations, delusions, suspiciousness or paranoia, irritability

11. Loss of impulse control (shown through undressing at inappropriate times or places or vulgar language)

12. An inability to carry out activities that involve multiple steps in sequence, such as dressing, making a pot of coffee, or setting the table

Behavior is the result of complex brain processes, all of which take place in a fraction of a second in the healthy brain. In AD, many of those processes are disturbed, and these disrupted communications between neurons are the basis for many distressing or inappropriate behaviors. For example, a person may angrily refuse to take a bath or get dressed because he does not understand what his caregiver has asked him to do. If he does understand, he may not remember how to do it. The anger can be a mask for his confusion and anxiety. Or, a person with AD may constantly follow her husband or caregiver and fret when the person is out of sight. To a person who cannot remember the past or anticipate the future, the world can be strange and frightening. Sticking close to a trusted and familiar caregiver may be the only thing that makes sense and provides security. The stages of AD spreading though the brain as in fig. 6

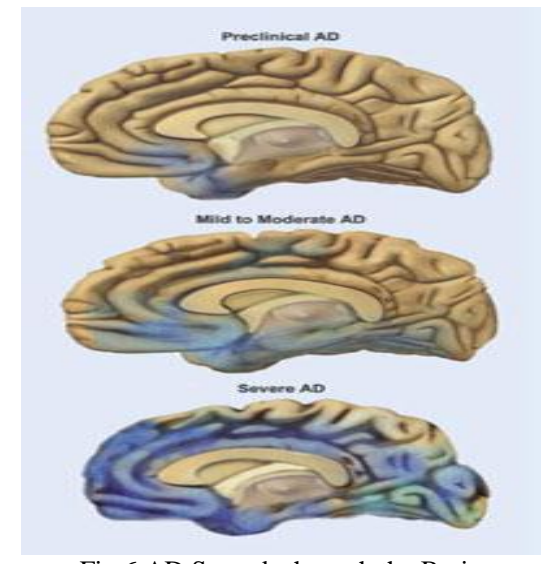

Fig.6 AD Spreads through the Brain

\section{Severe $A D$}

In the last stage of $\mathrm{AD}$, plaques and tangles are widespread throughout the brain, most areas of the brain have shrunk further, and ventricles have enlarged even more. People with $\mathrm{AD}$ cannot recognize family and loved ones or communicate in any way. They are completely dependent on others for care. Other symptoms can include:

1. Weight loss

2. Seizures

3. Skin infections

4. Difficulty swallowing 
5. Groaning, moaning, or grunting

6. Increased sleeping

7. Lack of bladder and bowel control

Near the end, the person may be in bed much or all of the time. The most frequent cause of death for people with AD is aspiration pneumonia. This type of pneumonia develops when a person is not able to swallow properly and takes food or liquids into the lungs instead of air. The brain shrinkage of cerebral cortex, Entorhinal cortex and hippocampus are shown in fig. 7.

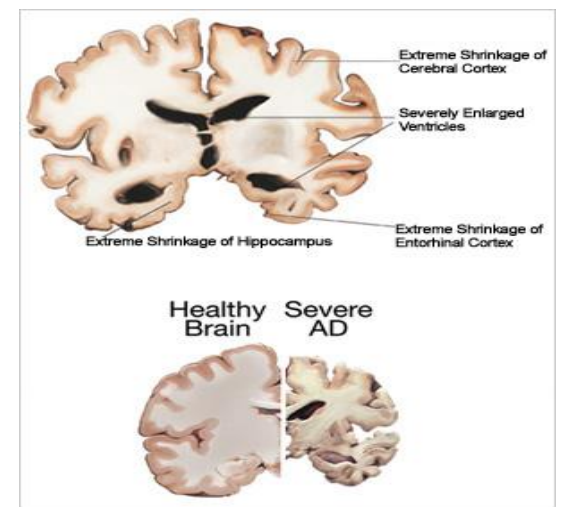

Fig. 7 Severe brain shrinkage of severe AD from healthy brain

\section{ALZHEIMER'S AND DEMENTIA TESTING FOR EARLIER DIAGNOSIS}

Current diagnosis of Alzheimer's disease is made by clinical, neuropsychological, and neuroimaging assessments. Routine structural neuroimaging evaluation is based on nonspecific features such as atrophy, which is a late feature in the progression of the disease. Therefore, developing new approaches for early and specific recognition of Alzheimer disease at the prodromal stages is of crucial importance.

\section{A. Biomarkers for earlier detection}

Current diagnosis of Alzheimer's relies largely on documenting mental decline. We now know that Alzheimer's has already caused severe brain damage in individuals who meet the criteria for mental decline. Researchers hope to discover an easy and accurate way to detect Alzheimer's before these devastating symptoms begin. Experts believe that biomarkers (short for "biological markers") offer one of the most promising paths. Biomarkers are reliable predictors and indicators of a disease process. Biomarkers include proteins in blood or spinal fluid, genetic variations (mutations) or brain changes detectable by imaging. Biomarkers must be "validated" their value as predictors and indicators must be confirmed by multiple studies in large groups of people. For example, high cholesterol is a validated biomarker for coronary heart disease. It is now accepted medical practice to monitor cholesterol and consider heart-healthy lifestyle changes or medications when blood levels exceed $200 \mathrm{mg} / \mathrm{dL}$ before a person develops active symptoms of heart disease [22].

\section{B. Brain Imaging/ neuroimaging}

Neuroimaging is among the most promising areas of research focused on early detection. Today, a standard workup for Alzheimer's disease often includes structural imaging with magnetic resonance imaging (MRI) or computed tomography (CT). These tests are currently used chiefly to rule out other conditions that may cause symptoms similar to Alzheimer's but require different treatment. Structural imaging can reveal tumors, evidence of small or large strokes, and damage from severe head trauma or a buildup of fluid in the brain. Preliminary research suggests that emerging imaging technologies and new applications of current technology may be able to detect hallmark changes associated with Alzheimer's disease in the brains of living individuals. If further research confirms the potential value of brain imaging, its use may one day be expanded to play a more direct role in diagnosing Alzheimer's and in earlier detection of the disease.

\section{Structural imaging}

The studies have shown that the brains of people with Alzheimer's, shrink significantly as the disease progresses. Research has also shown that shrinkage in specific brain regions such as the hippocampus may be an early sign of Alzheimer's. However, scientists have not yet agreed upon standardized values for brain volume that would establish the significance of a specific amount of shrinkage for any individual person at a single point in time.

\section{Functional imaging}

The research with positron emission tomography (PET) and other methods suggests that those with Alzheimer's typically have reduced brain cell activity in certain regions. For example, studies with fluorodeoxyglucose (FDG)-PET indicate that Alzheimer's disease is often associated with reduced use of glucose (sugar) in brain areas important in memory, learning and problem-solving. However, as with the shrinkage detected by structural imaging, there is not yet enough information to translate these general patterns of reduced activity into diagnostic information about individuals.

\section{Molecular imaging technologies}

These are among the most active areas of research aimed at finding new approaches to diagnose Alzheimer's in its earliest stages. Molecular strategies may detect biological clues indicating Alzheimer's is underway before the disease changes the brain's structure or function, or takes an irreversible toll on memory, thinking, and reasoning. Molecular imaging also may offer a new strategy to monitor disease progression and assess the effectiveness of nextgeneration, disease-modifying treatments. Molecular imaging compounds currently used in Alzheimer research include. 
Pittsburgh compound B (PIB) was the first radiotracer capable of highlighting deposits of beta-amyloid-one pathological hallmark of Alzheimer's disease-in living individuals during a PET scan. Imagine being able to see deep inside the brain tissue of a living person. If you could do that, you could find out whether the AD process was happening many years before symptoms were evident. This knowledge could have a profound impact on improving early diagnosis, monitoring disease progression, and tracking response to treatment.

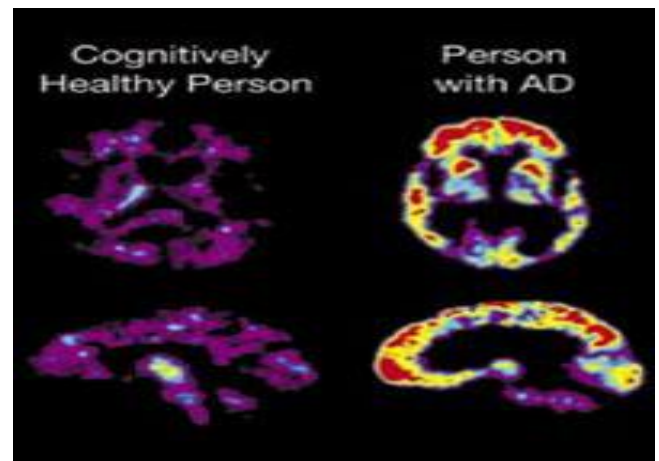

Fig.8 In this PET scan, the red and yellow colors indicate that PiB uptake is higher in the brain of the person with $\mathrm{AD}$ than in the cognitively healthy person

Scientists have stepped closer to this possibility with the development of a radiolabeled compound called Pittsburgh Compound $\mathrm{B}(\mathrm{PiB})$. PiB binds to beta-amyloid plaques in the brain, and it can be imaged using PET scans. Initial studies showed that people with $\mathrm{AD}$ take up more $\mathrm{PiB}$ in their brains than do cognitively healthy older people. Since then, scientists have found high levels of $\mathrm{PiB}$ in some cognitively healthy people, suggesting that the damage from beta-amyloid may already be underway. The next step will be to follow these cognitively healthy people who have high $\mathrm{PiB}$ levels to see whether they do, in fact, develop AD over time [23]. The other radiotracers used for PET scan are as follows

1. $18 F$ flutemetamol (flute) another radiotracer that highlights beta-amyloid in a PET scan, is structurally identical to PIB except for one fluorine atom in place of a carbon atom. That small chemical change enables flutemetamol to remain stable significantly longer than does PIB, potentially increasing its usefulness outside research settings.

2. Florbetapir $F 18(18 F-A V-45)$ is also a radiotracer that highlights brain beta-amyloid during a PET scan. The developer has sought Food and Drug Administration (FDA) approval to market florbetapir under the brand name Amyvid. The FDA has said it will withhold approval until the developer establishes a professional training program to ensure accuracy and consistency in reading and interpreting Amyvid scans.

3. Florbetaben (BAY 94-9172) is another radiotracer designed to detect beta-amyloid during a PET scan.

\section{DISCUSSION}

Even though there are different neuropathology related tests, various imaging modalities, biomarkers and drug therapies etc for the diagnosis of $\mathrm{AD}$, they are insufficient for a definite diagnosis. But if we can combine the features of all of the above using soft computing techniques such as fuzzy logic, neural computing, evolutionary computation, and probabilistic reasoning, it may be possible to early diagnosis of the disease in a convenient way. Soft computing differs from conventional (hard) computing in that, unlike hard computing, it is tolerant of imprecision, uncertainty, partial truth, an approximation. In effect, the role model for soft computing is the human mind. The guiding principle of soft computing is: "Exploit the tolerance for imprecision, uncertainty, partial truth, and approximation to achieve tractability, robustness and low solution cost". The clinical data may consist of missing, incorrect and sometimes incomplete values set so using soft computing is the better alternative to handle such data. The principal constituent methodologies in soft computing are complementary rather than competitive. Fuzzy logic handles imprecision, neural computing deals with learning, evolutionary computation is for optimization and probabilistic reasoning handles uncertainty.

\section{CONCLUSION}

Alzheimer disease (AD) is a fast progressive and irreversible disease which affects the whole brain function. Although there are many clinical treatments and neuroimaging techniques which slower $\mathrm{AD}$, they are not at all effective for the early diagnosis. However, despite comprehensive searches, no single test has shown adequate sensitivity or specificity, and it is likely that a combination will be needed. As the complexity of this diagnostic process increases, so does the vital importance of ensuring consistent and accurate laboratory assessment. This paper gives a clear idea about the different causes and the strategies used for the early diagnosis of AD.

\section{REFERENCES}

[1] D. S. Knopman, J. Kitto, S. Deinard and J. Heiring, "Longitudinal study of death and institutionalization in patients with primary degenerative dementia", J Am GeriatrSoc, Vol. 36, pp.108-112, 1988.

[2] J. L. Cummings, H. V. Vinters, G. M. Cole and Z. S. Khachaturian, "Alzheimer's disease: etiologies, pathophysiology, cognitive reserve and treatment opportunities", Neurology, Vol. 51, pp. 2-17, 1998.

[3] C. S. Sandeep and A. Sukesh Kumar, "The Early Confirmation of Alzheimer's Disease using Internet Sources", Asian Journal of Science and Applied Technology", Vol. 6, No. 1, pp.10-17, 2017.

[4] C. Wolfson, D. B. Wolfson, M. Asgharian, C. E. M'Lan, T. Østbye, K. Rockwood and D. B. Hogan, "A reevaluation of the duration of survival after the onset of dementia", N Engl J Med, Vol. 344, No. 15, pp.1111-6, 2001.

[5] G. K. Wilcock and M. M. Esiri "Plaques, tangles, and dementia. A quantitative study”, J NeurolSci, Vol. 56, pp.343-56, 1982.

[6] C. S. Sandeep, A. Sukesh Kumar, K. Mahadevan and P. Manoj, "Analysis of MRI and OCT Images for the Early Diagnosis of Alzheimer's Disease Using Wavelet Networks", AMSE journal on Lectures on Modelling and Simulation, Vol. 1, pp. 31-40, 2018. 
[7] P. V. Arriagada, J. H. Growdon, E. T. Hedley-Whyte and B. T. Hyman, "Neurofibrillary tangles but not senile plaques parallel duration and severity of Alzheimer's disease", Neurology, Vol. 42, No. pp. 631-9, 1992.

[8] C. S. Sandeep, A. Sukesh Kumar, K. Mahadevan and P. Manoj, "Analysis of Retinal OCT Images for the Early Diagnosis of Alzheimer's Disease", Springer-Advances in Intelligent Systems and Computing book series (AISC), Vol. 749, pp. 509-520, 2018.

[9] M. A. Westerman, D. Cooper-Blacketer, A. Mariash, L. Kotilinek, T. Kawarabayashi, L. H. Younkin, G. A. Carlson, S. G. Younkin and K. $\mathrm{H}$. Ashe "The relationship between $\mathrm{A} \beta$ and memory in the $\mathrm{Tg} 2576$ mouse model of Alzheimer's disease ", Journal of Neuroscience, Vol. 22, No. 5, pp. 1858-67, 2002.

[10] A. Ott, R. P. Stolk, F. Van Harskamp, H. A. P. Pols, A. Hofman and M. M. B. Breteler "Diabetes mellitus and the risk of dementia: The Rotterdam study", Neurology, Vol.53, No.9, pp.1937-42,1999.

[11] I. Skoog, B. Lernfelt, S. Landahl, B. Palmertz, L-Andreasson, L. Nilsson, G. Persson, A. Oden and A. Svanborg., "15- year longitudinal study of blood pressure and dementia.”, Lancet, Vol.347, pp.141-5, 1996.

[12] C. S. Sandeep and A. Sukesh Kumar, "A Psychometric Assessment Method for the Early Diagnosis of Alzheimer's disease", International Journal of Scientific \& Engineering Research -IJSER, Vol.8 No.3, pp.901-905, 2017

[13] D. A. Snowdon, S. J. Kemper, J. A. Mortimer, L. H. Greiner, D. R. Wekstein and W. R. Markesbery "Linguistic ability in early life and cognitive function and Alzheimer's disease in late life: Findings from the nun study", J Am Med Assoc., Vol.275, No.7, pp.528-32, 1996

[14] R. Yaari, and J. Corey-Bloom, "Alzheimer's disease: Pathology andpathophysiology”, Semin Neurol., Vol. 27, pp. 32-41, 2007.
[15] C. S. Sandeep and A. Sukesh Kumar, "A Review on the Early Diagnosis of Alzheimer's Disease (AD) through Different Tests, Techniques and Databases", AMSE Journals: Modelling C, Vol. 76; No. 1; pp 1-22, 2015

[16] E. B. Larson, L. Wang and J. D. Bowen, "Exercise is associated withreduced risk for incident dementia among persons 65 years of age andolder", Ann Intern Med., Vol.144, pp.73-81, 2006.

[17] R. J. Harvey, M. Skelton-Robinson and M. N. Rossor "The prevalence andcauses of dementia in people under the age of 65 years", JNeurolNeurosurg Psychiatry, Vol. 74, pp. 1206-9, 2003.

[18] L. A. Farrer, L. A. Cupples and J. L. Haines, "Effects of age, sex, and ethnicity on the association between apolipoprotein E genotype and Alzheimer disease, A meta-analysis", APOE and Alzheimer DiseaseMeta Analysis Consortium. JAMA, Vol. 278, pp. 1349-56, 1997.

[19] C. P. Ferri, M. Prince and C. Brayne, "Global prevalence of dementia: aDelphi consensus study", Lancet, Vol. 366, pp. 2112-7, 2005.

[20] C. S. Sandeep, A. Sukesh Kumar and M. J. Susanth, "The Online Datasets Used to Classify the Different Stages for the Early Diagnosis of Alzheimer's Disease (AD)", International Journal of Engineering and Advanced Technology, Vol. 6, No. 4, pp. 38-45, 2017

[21] H. F. Chiu, L. C. Lam and I. Chi, "Prevalence of dementia in Chineseelderly in Hong Kong", Neurology, Vol.50, pp.1002-9, 1998

[22] L. X. Hy and D. M. Keller, "Prevalence of AD among whites: a summary bylevels of severity", Neurology, Vol. 55, pp. 198-204, 2000.

[23] L. W. Chu, S. Tam and R. L. Wong, "Bioavailable testosterone predicts alower risk of Alzheimer's disease in older men", $J$ Alzheimers Dis, Vol. 21, pp. 1335-45, 2010. 